\title{
Implementation of a breast cancer genetic service in South Africa - lessons learned
}

\author{
M Schoeman, ${ }^{1}$ MSc (Med) (Genet Counsell), BSc (Hons) (Genet), BSc; J P Apffelstaedt, ${ }^{2}$ FCS (SA); K Baatjes, ${ }^{2}$ FCS (SA); \\ M Urban, ${ }^{1}$ FCPaed (SA) \\ ${ }^{1}$ Division of Molecular Biology and Human Genetics, Faculty of Medicine and Health Sciences, Stellenbosch University and Tygerberg Hospital, \\ Cape Town, South Africa \\ ${ }^{2}$ Department of Surgery, Stellenbosch University and Breast Clinic, Tygerberg Hospital, Cape Town, South Africa
}

Corresponding author: M Schoeman (mardelle@sun.ac.za)

Background. Genetic testing for BRCA mutations has been available in the Western Cape of South Africa since 2005, but practical implementation of genetic counselling and testing has been challenging.

Objective. To describe an approach to breast cancer genetic counselling and testing developed in a resource-constrained environment at Tygerberg Hospital in Cape Town, Western Cape.

Methods. Genetic counselling is offered in a stepwise manner to our diverse patient population, with a focus on affected probands, and subsequent cascade testing. A record review of BRCA testing between 2005 and 2011 was performed.

Results. During this period 302 probands received genetic testing, with increasing numbers tested over time. Of 1520 women treated for breast cancer since 2008, 226 (14.9\%) accepted BRCA testing, and 39 tested positive (17.3\% of those tested, and $2.6 \%$ of all women). Common founder mutations were detected in $11.9 \%$ of women (36/302), and comprised 73\% (36/49) of mutations detected. Cascade testing increased after 2010: 16 female and 4 male family members of 19 probands accepted testing, with 6 positives being detected.

Conclusion. A protocol-driven approach focusing on probands, with initial pre-test counselling by primary care staff has proven effective in establishing the service. Involvement of a clinical geneticist/genetic counsellor has permitted more detailed post-test counselling and increased use of cascade testing.

S Afr Med J 2013;103(8):529-533. DOI:10.7196/SAMJ.6814

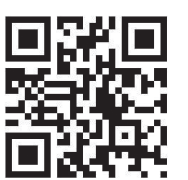

The most recent available national estimates regarding the frequency of breast cancer diagnosis in South African (SA) women indicates a 1 in 29 lifetime risk of developing breast cancer. ${ }^{[1]}$ The incidence of breast cancer in SA varies by time and place, but there is evidence that it is becoming more common. ${ }^{[2]}$ In the Western Cape Province (WCP), breast cancer is relatively common, accounting for $3 \%$ of all mortality in adult women in Cape Town - a higher percentage than for any other cancer. ${ }^{[3]}$ Breast cancer is associated with a high mortality rate in SA, mainly as a result of delayed diagnosis, ${ }^{[4]}$ for reasons including limited community awareness and restricted access to oncology care facilities. ${ }^{[5]}$

A small but important minority of breast cancer cases are due to mutations in the BRCA1 and 2 genes, often referred to as hereditary breast and ovarian cancer (HBOC). Genetic counselling and testing for women with a family history of breast cancer has been available for well over a decade, and guidelines exist for the genetic counselling and testing process. ${ }^{[6]} \mathrm{HBOC}$ has sufficient clinical and public health relevance that the US Preventive Services Task Force recommends programmes for genetic counselling and testing of HBOC. ${ }^{[7]}$

In SA, diagnostic BRCA testing has been available since 2005, and has been facilitated by the identification of common mutations in the BRCA1 and 2 genes in both Afrikaner ${ }^{[8,9]}$ and other local populations. ${ }^{[10]}$ This has allowed genetic counselling and testing to be offered, but attempts to establish programmes for genetic counselling and testing have faced many challenges. These include: the relative complexity of the risk assessment and testing approach; limited financial and human resources; limited community knowledge of breast cancer or of the possibility of a familial cancer; and difficulty accessing old hospital records of family members.

The translation of genomic research into routine practice is inherently complex. Its lengthy translational research process was described by Khoury et al. ${ }^{[11]}$ in 2007 . However, little is known about this process beyond the relatively resource-rich infrastructures in which it usually takes place. ${ }^{[12]}$ We therefore describe a programme for genetic counselling and BRCA testing developed in a resourceconstrained environment, how it evolved over time and the rates of BRCA mutation detection and family follow-up achieved.

\section{Materials and methods Population}

The population of the WCP of SA is diverse, comprising several predominant groups. Black South Africans of mainly Xhosa ethnicity make up a quarter of the population, whites of Afrikaner and non-Afrikaner ancestry a quarter, and people of mixed ancestry approximately half of the population. Tygerberg Hospital provides tertiary-level public health services to the eastern half of the Cape Town metropole, as well as half of the rural population of the WCP, with a total referral population of roughly 2.5 million people. Tygerberg Hospital is a public health facility and, as such, serves the $70 \%$ of people who do not have access to private healthcare facilities, a predominantly 'working poor' and indigent population. Women (and men) suspected of having breast cancer are referred to the Tygerberg Hospital breast cancer team from throughout the region for diagnosis and management. 


\section{Genetic counselling and testing}

Throughout the time period, the focus was on identifying and offering genetic testing to those assessed as being at high risk of HBOC. The criteria for testing had been developed prior to 2005 by the local breast surgeons in collaboration with a molecular genetic laboratory researching HBOC, based elsewhere in SA. The initial criteria for offering BRCA testing were that the woman should be a proband affected by breast cancer with at least one additional risk factor on personal or family history.

Personal risk factors included: $(i)$ age $<40$ years; (ii) bilateral breast cancer; and (iii) additional ovarian cancer.

Family history factors included: (i) 1 firstdegree relative with breast or ovarian cancer; (ii) $>1$ second-degree relatives affected; and (iii) male breast cancer in a relative.

The process for genetic counselling and testing in the period of 2005 - 2009 is depicted in Fig. 1. The multidisciplinary breast cancer team, comprising surgeons and oncologists, primarily provided genetic counselling (both pre- and post-test). Due to human resource capacity constraints, involvement of the clinical geneticist was by referral only, for patients testing positive. The hospital did not have a genetic counsellor employed during this period. Genetic testing comprised testing for mutations that are relatively common in the population (founder mutations), including:

- 3 mutations which make up $>90 \%$ of BRCA mutations in Afrikaners - BRCA1 1493delC, BRCA1 2760G>T (p.Glu881X) and BRCA2 8162delG. ${ }^{[8,9]}$

- the 3 mutations known to be common in those of Ashkenazi Jewish ancestry BRCA1 185delAG, BRCA1 1493insC and BRCA2 6174delT.

- a recently described mutation that has been found to be more common in people of Xhosa and mixed ancestry in the WCP (BRCA2 5999del4) was added to the panel of 'common mutations' in 2009. ${ }^{[10]}$

If the common mutations were excluded and there was a family history of breast cancer, the laboratory would add a protein truncation test for exon 11 of the BRCA1 gene and exon 10 and 11 of the BRCA2 gene.

The genetic counselling and testing process was reviewed jointly by the breast surgeons and clinical geneticists at the beginning of 2010 and altered (as depicted in Fig. 2), to increase the role of the clinical geneticist/genetic counsellor. The aim of this was to: (i) improve post-test counselling; (ii) ration more expensive 'full' BRCA screening tests, which had recently become available - these include full sequencing of all exons of both the BRCA1 and 2 genes, as well as multiplex ligation probe analysis (MLPA) for large rearrangements; and (iii) improve family communication and follow-up for BRCApositive cases. Thus, a clinical geneticist/genetic counsellor became a regular participant in the multidisciplinary team, reviewed all results, and provided post-test genetic counselling (which

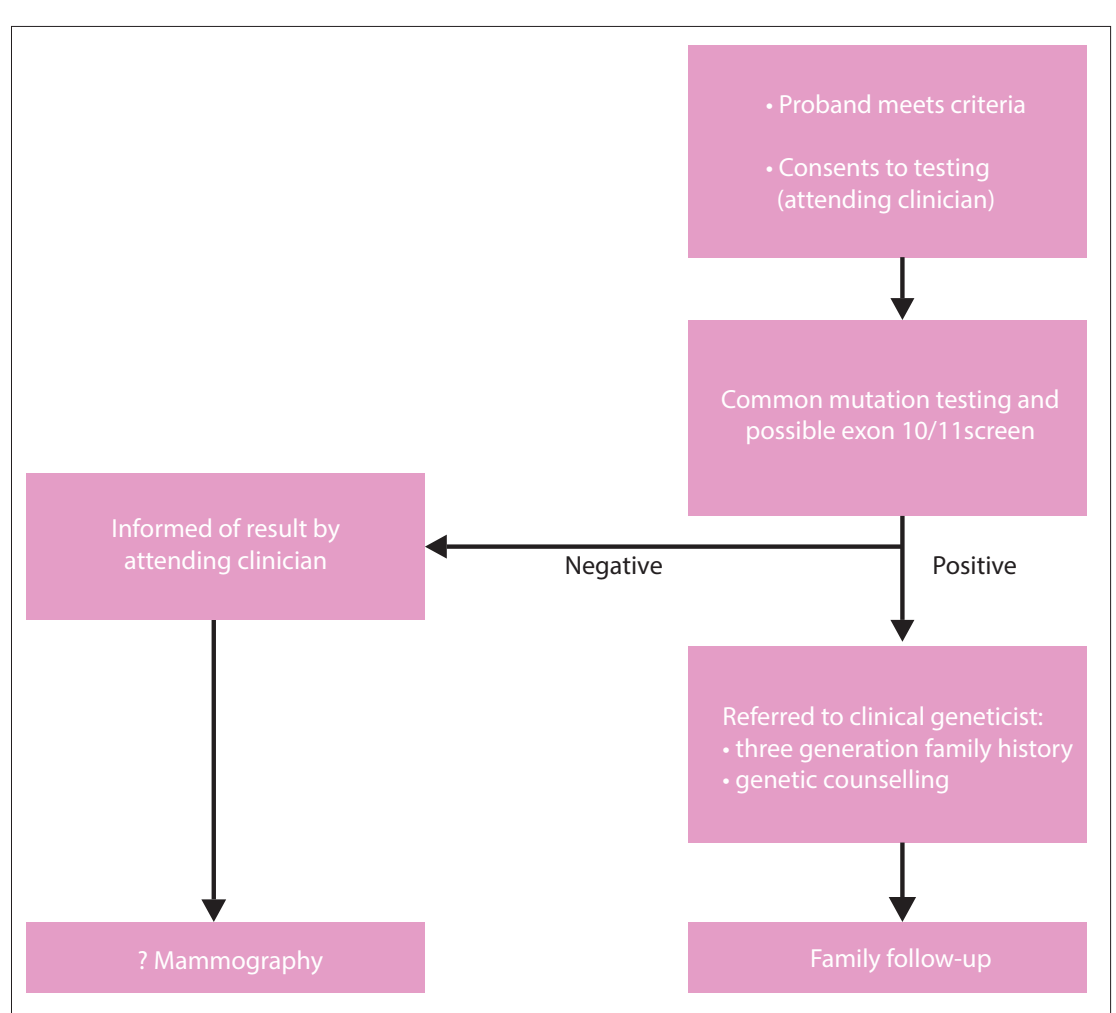

Fig. 1. Counselling and testing process, 2005 - 2009.

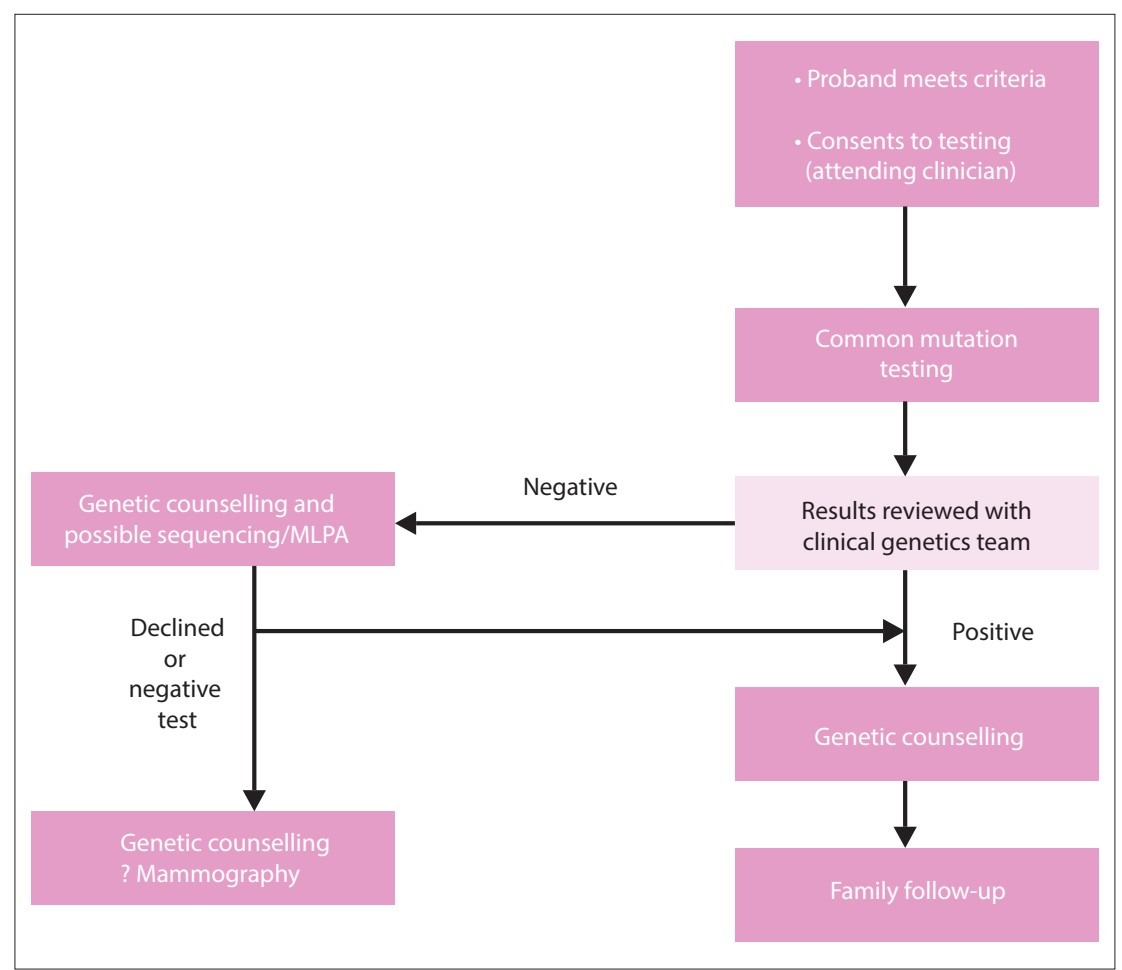

Fig. 2. Counselling and testing process, 2010 - 2011. included a discussion on further testing as appropriate), and provided cascade screening to

Genetic counselling, including a threegeneration family history, was conducted prior to the offer of full BRCA screening, which was offered to women with breast family members. 
cancer who met any one of the following personal or family history criteria:

- Personal risk factors: (i) breast cancer at $<35$ years, (ii) bilateral breast cancer at $<70$ years, and (iii) additional ovarian cancer at any age.

- Family history factors: $(i)$ at least 1 first-degree relative with breast cancer at $<50$ years or ovarian cancer at any age, and (ii) male breast cancer in a relative.

\section{Record review}

A review of laboratory records was conducted for all BRCA tests performed in women from the time of initiation of the programme in 2005 until December 2011. For patients testing BRCA-positive, hospital records were obtained and assessed. The following data were obtained: BRCA tests performed and results thereof, patient's age at test (which was usually performed early in the course of patient management), ethnic background (where available) and family history.

\section{Statistical analysis}

Univariate analysis of categorical data was conducted using the twotailed Fischer exact test, and 95\% confidence intervals (CIs) calculated using the modified Wald method.

\section{Ethics approval}

Approval for the audit was obtained from the Research Ethics Committee of the University of Stellenbosch (no. N10/11/381). A waiver of informed consent was obtained.

\section{Results}

The number of genetic tests performed per year is described in Table 1. Denominator data were only available from 2008. Since then, there have been 1520 patients newly diagnosed with breast cancer, of whom 39 (2.6\%; CI 1.8\% - 3.4\%) tested positive for BRCA1 or BRCA2 mutations, and 27 (1.8\%; CI 1.2\% - 2.6\%) carried 'common mutations'.

The performance of the individual genetic tests in detecting BRCA mutations is described in Table 2. There was a slight overlap of the tests used in the different time periods. Of 131 protein truncation tests performed, 125 were prior to 2010 , and $80 \%$ of the probands went on to have a protein truncation test (125/157 probands who tested negative for common mutations). Prior to 2010, only 3 individuals went on to sequencing of selected BRCA1/2 exons. Beginning in 2010, 29 individuals received full sequencing rather than protein truncation testing, and if negative, went on to MLPA testing. This comprised 20\% of the 145 probands who tested negative for common mutations. Variants of unknown significance were identified in 7/29 full sequencing cases, the majority of which were novel variants. The mutations detected are detailed in Table 3. Note that testing for the BRCA2 5999del4 mutation was introduced only in 2009.
The ethnic group distribution amongst BRCA mutation-positive women is described in Table 4. The proportion of mutations detected in the white and mixed-ancestry population group is similar; although considerably more mixed ancestry than white women are treated at the clinic (exact figures are not available since we do not maintain ethnicity data for the entire clinic population). Black African women accounted for only $10.2 \%$ of mutation-positive cases. Although this number appears small, it is important to note that women of black African (mainly Xhosa) origin accounted for only 24/302 (7.9\%) cases tested for BRCA mutations between 2005 and 2011.

Follow-up of family members was compared before and after the clinical geneticist/genetic counsellor was included in the multidisciplinary team. Before this time, there were 30 BRCA mutations detected in probands. Only 2 family members reached the clinical geneticist for genetic counselling, whereas a total of 4 received BRCA testing ( 0.13 per proband) and 2 of these tested positive. Notably, following inclusion of the clinical geneticist/genetic counsellor, 19 probands had a positive BRCA test; 20 family members (1.05 per proband) received genetic counselling, all of whom consented to genetic testing and 6 of whom tested positive.

\section{Discussion}

\section{Genetic counselling and testing approach}

An important starting point for our service was to focus on women affected with breast cancer rather than the 'worried well. This strategy arose naturally from the initial research project on genetic testing for hereditary breast cancer, but has proved to be important in establishing an ongoing role for genetic counselling and testing. It efficiently targets genetic counselling at those eligible for testing. It avoids targeting the

Table 1. Female breast cancer incidence and BRCA testing by year

\begin{tabular}{llll}
\hline Year & $\begin{array}{l}\text { New cases } \\
\boldsymbol{n}\end{array}$ & $\begin{array}{l}\text { BRCA tests performed } \\
\boldsymbol{n} \text { (\% new cases) }\end{array}$ & $\begin{array}{l}\text { Positive tests } \\
\boldsymbol{n} \text { (\% new cases) }\end{array}$ \\
\hline 2005 & - & 5 & $1(20)$ \\
2006 & - & 33 & $3(9)$ \\
2007 & - & 38 & $6(15.8)$ \\
2008 & 338 & $37(11)$ & $5(13.5)$ \\
2009 & 367 & $60(16.3)$ & $15(25)$ \\
2010 & 368 & $58(15.8)$ & $8(13.8)$ \\
2011 & 447 & $71(15.9)$ & $11(15.5)$ \\
Total, $N(\%)$ & $1520^{*}$ & $226^{*} / 302$ & $39^{*} / 49(16.2)$ \\
${ }^{*}$ Since 2008. & & &
\end{tabular}

Table 2. BRCA tests and detection rates from 2005 - 2011

\begin{tabular}{lll}
\hline Genetic test & $\begin{array}{l}\text { Individuals tested } \\
\boldsymbol{n}\end{array}$ & $\begin{array}{l}\text { Individuals testing positive } \\
\boldsymbol{n}(\%)\end{array}$ \\
\hline $\begin{array}{l}\text { First line } \\
\quad \text { Targeted analysis for common mutations }\end{array}$ & 302 & $36(11.9)$ \\
Second line & & $9(6.9)$ \\
$\quad$ Protein truncation test of exon $10 / 11$ & 131 & $4(13.8)$ \\
$\quad$ Full sequencing of BRCA1/2 genes & 29 & $0(0)$ \\
$\quad$ MLPA for rearrangements of BRCA1/2 genes & 25 & $49(16.2)$ \\
Total, $N$ (\%) & 302 & -12.6 \\
CI $=$ confidence interval; MLPA multiplex ligation probe analysis. & & -
\end{tabular}


Table 3. Description of BRCA mutations detected

\begin{tabular}{lll}
\hline Genetic test & Mutation & $\begin{array}{l}\text { Mutations detected } \\
\boldsymbol{n}(\%)\end{array}$ \\
\hline First line & & \\
Targeted mutation analysis & BRCA2 8162delG & $17(34.7)$ \\
& BRCA2 5999del4 & $7(14.3)$ \\
& BRCA1 p.E881X & $7(14.3)$ \\
& BRCA1 185delAG & $2(4.1)$ \\
& BRCA1 1493delC & $2(4.1)$ \\
& BRCA1 5383insC & $1(2.0)$ \\
Total & BRCA2 6174delT & $0(0.0)$ \\
Second line & & $36(73.5)$ \\
Protein truncation test of exon 10/11 & & \\
Sequencing & BRCA1 & $4(8.2)$ \\
& BRCA2 & $5(10.2)$ \\
MLPA & BRCA1 & $2(4.1)$ \\
Total & BRCA2 & $2(4.1)$ \\
BRCA1, $N$ (\%; CI) & & $0(0.0)$ \\
BRCA2, $N$ (\%; CI) & & $13(26.5)$ \\
Total, $N$ (\%) & & $18(36.7 ; 23.4-51.7)$ \\
MLPA = multiplex ligation probe analysis; CI = confidence interval. & $31(63.3 ; 48.3-76.6)$ \\
& & $49(100)$ \\
& &
\end{tabular}

Table 4. Ethnic group distribution amongst BRCA mutation-positive women

\begin{tabular}{lll}
\hline & $\begin{array}{l}\text { Mutation-positive } \\
\boldsymbol{n} \text { (\% of total mutations) }\end{array}$ & $\begin{array}{l}\text { Common mutations } \\
\boldsymbol{n} \text { (\% of total mutations in ethnic group) }\end{array}$ \\
\hline White (mainly Afrikaner) & $21(42.9)$ & $18(85.7)$ \\
Mixed ancestry & $23(46.9)$ & $14(60.9)$ \\
African & $5(10.2)$ & $4(80)$ \\
Total, $N$ & 49 & 36
\end{tabular}

worried well, which is wasteful, given the current low level of awareness of familial breast cancer in our community and the difficulties in tracing affected family members.

Implementation of genetic testing has also been facilitated by a stepwise approach to testing, in which consent is initially obtained only for testing of common mutations. This step is relatively inexpensive and provides results that are easy to interpret. Initial pre-test counselling is provided by a primary care professional (surgery or oncology staff members) early in the course of treatment for breast cancer, using a checklist to determine eligibility for testing. The need for this approach arose as a direct consequence of the scarcity of genetics-trained professionals in our local environment.

The use of simple criteria to identify women eligible for the initial offer of testing has facilitated its implementation, and is

- the relatively low cost of common mutation testing makes it possible to offer it to a broader group of women

- more sophisticated scoring systems designed to estimate the likelihood of BRCA carrier status ${ }^{[13]}$ are cumbersome and of uncertain relevance in our patient population

- family history information is often limited. Because the concept of cancer is unfamiliar to many patients, communication between family members may not include the cause of death of relatives, and may not permit identification of the particular cancer causing the death.

Involvement of the primary care staff in the counselling process maintains their stake in the appropriate in our setting because: genetic counselling and testing programme, and is consistent with a multidisciplinary team approach. Initiation of counselling and testing by a primary care professional is aimed at maximising access to genetic testing, and has resulted in $14.9 \%$ uptake of common mutation testing (since 2008: 226/1 520 breast cancer cases were tested). We were unable to find information on the proportion of breast cancer patients receiving BRCA testing in other populations, but feel that our apparently high rate of common mutation testing is justified by its relatively low cost and an acceptable mutation detection rate of $11.9 \%$.

Concerns have been raised regarding the appropriateness of genetic counselling and testing being performed shortly after diagnosis, in view of the potential psychosocial consequences. ${ }^{[14]}$ However, this concern is mitigated by accumulating evidence of the benefit of tailoring surgery and adjuvant chemotherapy according to mutation status. ${ }^{[15]}$ Treatmentfocused genetic testing is used in women newly diagnosed with cancer to provide them, as well as their managing clinicians, with important information on which treatment decisions can be based. ${ }^{[16]}$ It is also used in our setting for reducing the cost and discomfort of recurrent surgeries. A recent study found that the benefits of knowing one's BRCA mutation status early outweighed the potential psychosocial harms caused by learning this result shortly after being diagnosed. ${ }^{[16]}$

Another reason for initiating BRCA testing early in the course of treatment is the fact that breast cancer has a relatively poor prognosis in our setting (as in many developing countries), particularly for younger women, ${ }^{[17]}$ who are most likely to be eligible for testing. If BRCA testing is not considered timeously, the opportunity may be lost altogether. Although testing in these circumstances may not be to the benefit of the affected individual, it is important to be aware of the unaffected family members' interest in genetic information. ${ }^{[15]}$

As capacity for genetic counselling improved over time, we chose to prioritise genetic counsellor involvement in post-test counselling for common mutation testing, which included decision-making regarding 'full' BRCA screening. This step involves more complex information, more expensive testing, and the feedback of potentially difficult results. The implementation of post-test counselling by a genetic counsellor has allowed us to review whether any harm has arisen from the initial offer of testing occurring early in the course of disease by a primary care professional. Our experience is that there have not been any obvious adverse effects to date, at least when this approach is accompanied by detailed post- 
test counselling by a genetic counsellor. Importantly, as both the genetic counselling capacity has improved and with integration of the counsellor within the multidisciplinary team, it has been possible to increase pretest counselling provided by the genetic counsellor.

Until post-test counselling was routinely provided by a genetic counsellor, cascade testing proved very difficult to implement. Improved access to a genetic counsellor has resulted in greater numbers of family members presenting for genetic counselling, and they appear to be a self-selected group who all want genetic testing. Depending on their place of residence, family members may present themselves to another breast cancer service in Cape Town or further afield, but we are not able to ascertain these numbers. Family members found to carry BRCA mutations are referred for discussion of management and surveillance options. At this stage we have not assessed how family members have used the knowledge of their BRCA status.

\section{BRCA mutation detection rates}

The relatively high detection rate for common mutations in our clinic (representing $1.8 \%$ of all women with breast cancer, $11.9 \%$ of those tested, and $73 \%$ of all mutations detected) underlines the value of first testing for these mutations. Although we serve an ethnically diverse population, common mutations exist in at least 2 large groups of patients - Afrikaners and black South Africans of Xhosa ethnicity while common mutations from both groups occur in those of mixed ancestry.

For practical reasons, testing for 'common mutations' includes a standard panel of mutations, irrespective of the patient's ancestry. This panel has enlarged over time from 3 mutations to 7 mutations as new information has become available; it is possible that further mutations will be added as knowledge increases regarding the BRCA variants in black and mixed-ancestry South Africans. Two disease-causing variants in the BRCA2 gene (the BRCA2 8162delG, and the BRCA2 5999del4 mutations) are very common and result in considerably more BRCA2-positive individuals being diagnosed than BRCA1 in our patient population, the reverse of the situation described in Western populations. The relatively small number of black African women tested reflects the current relatively low prevalence of breast cancer in this group, as well as the fact that BRCA testing in this group became more relevant following the inclusion of the BRCA2 5999del4 mutation in the common mutation panel after 2009.

Further analysis by protein truncation testing of large exons, as applied in our programme, had a relatively low detection rate. Although more costly, and therefore used more sparingly, sequencing of the BRCA genes appears to have a relatively good detection rate and added significantly to the targeted analysis for common mutations. Given the difference in cost of the 2 methods, the cost-effectiveness of the 2 approaches may be similar. Among 25 patients receiving MLPA testing, no large genetic rearrangements were detected. Variants of unknown significance were identified in a high proportion of cases receiving full sequencing $(7 / 29 ; 24 \%)$, posing a significant counselling challenge. Further characterisation of these variants should be an important area of research in our population in the future.

Extrapolating back to the population of breast cancer patients, we found an overall rate of BRCA mutations of $2.6 \%$. This represents a minimum prevalence given the targeted rather than comprehensive testing strategy we used. Although there is considerable variation in the frequency of BRCA mutations between different populations, our detection rate is comparable with rates determined in comprehensive research studies in some Western populations. ${ }^{[18]}$ This suggests that either our testing programme detects a high proportion of all BRCA mutations or, more likely, that the rate of BRCA positivity is high in all or part of the population we serve as a result of founder effects.

\section{Recommendations for future research}

The impact of receiving pre-test counselling for founder mutations from a primary care staff member, rather than a genetic counsellor, requires formal investigation. Further studies should also determine how knowledge of BRCA status has been used in family members found to have a BRCA1 or 2 mutation.

\section{Study limitation}

The study comprised a retrospective analysis of a relatively limited number of available data fields. However, by restricting the number of data fields we were able to ensure that all information was available for almost all patients. Although our approach may be generalisable, it should be noted that the utility of common mutation testing might vary in different parts of SA.

\section{Conclusions}

Breast cancer genetic counselling programmes to stratify patients for possible genetic testing are difficult to implement in the SA environment, due to resource limitations and other challenges. A limited number of mutations are common in subsets of our diverse population, with overlaps in patients of mixed ancestry. This reality has facilitated genetic counselling and testing. The programme has also been facilitated by a pragmatic approach, with pre-test counselling for common mutation testing being undertaken by a member of the breast cancer team relatively soon after diagnosis, and post-test counselling being provided by a clinical geneticist or genetic counsellor to ensure that patients are appropriately informed. Family follow-up has increased considerably with the involvement of a genetic counsellor.

\section{References}

1. South African National Cancer Registry. National Cancer Registry Tables 2001. http://www.cansa.org.za/ (accessed 25 March 2012).

2. Fregene A, Newman, LA. Breast cancer in sub-Saharan Africa: How does it relate to breast cancer in African American women? Cancer 2005;103(8):1540-1550. [http://dx.doi.org/10.1002/cncr.20978] . Groenewald P, Bradshaw D, Daniels J, et al. Cause of death and premature mortality in Cape Town, 20012006. Cape Town: Medical Research Council, 2008. http://www.sahealthinfo.org/bod/causeofdeath2008. pdf (accessed 25 March 2012)

4. Murray EM. Medical and radiation oncology for breast cancer in developing countries with particular reference to locally advanced breast cancer. World J Surg 2003;27(8):924-927. [http://dx.doi.org/10.1007/ s00268-003-6977-9]

5. Vorobiof DA, Sitas F Vorobiof G. Breast cancer incidence in South Africa.J Clin Oncol 2001:19(S18):125S-127S.

6. Robson ME, Storm CE, Weitzel J, Wollins DS, Offit K. American Society of Clinical Oncology policy statement update: Genetic testing for cancer susceptibility. J Clin Oncol 2010;28(5):893-901. [http://dx.doi statement update: Genetic testin

org $/ 10.1200 / / \mathrm{CO} .2009 .27 .0660]$
US Preventive Services Task Force. Genetic risk assessment and BRCA mutation testing for breas US Preventive Services Task Force. Genetic risk assessment and BRCA mutation testing for breast
and ovarian cancer susceptibility: Recommendation statement. Ann Intern Med 2005;143(5):355-361. [http://dx.doi.org/10.7326/0003-4819-143-5-200509060-00011]

8. Reeves MD, Yawitch TM, van der Merwe NC, et al. BRCA1 mutations in South African breast and or ovarian cancer families: Evidence of a novel founder mutation in Afrikaner families. Int J Cancer 2004;110(5):677-682. [http://dx.doi.org/10.1002/ijc.20186]

9. Van der Merwe NC, van Rensburg EJ. Hereditary breast/ovarian cancer and BRCA mutations: A South African perspective. Abstract, Third International Symposium on Hereditary Breast and Ovarian Cancer. Quebec, Canada 14 - 16 October 2009. Current Oncology 2009;16(5):91-110.

10. Van der Merwe N, Hamel N, Schneider SR, et al. A founder BRCA2 mutation in non-Afrikaner breast cancer patients of the Western Cape of South Africa. Clin Genet 2012;81(2):179-184. [http://dx.doi. cancer patients of the Western Cape of
org $/ 10.1111 / j .1399-0004.2010 .01617 . x]$

11. Khoury MJ, Gwinn M, Yoon PW, et al. The continuum of translation research in genomic medicine: How can we accelerate the appropriate integration of human genome discoveries into health care and disease prevention? Genet Med 2007;9(10):665-674. [http://dx.doi.org/10.1097/GIM.0b013e31815699d0]

prevention? Genet Med 2007;9(10):665-674. [http:///dx.doi.org/10.1097/GIM.0b013e31815699d0]
12. Kerner J, Rimer B, Emmons, K. Introduction to the special section on dissemination: Dissemination Kerner J, Rimer B, Emmons, K. Introduction to the special section on dissemination: Dissemination
research and research dissemination: How can we close the gap? Health Psychol 2005;24(5):443-446. research and research dissemination: How can
[http://dx.doi.org/10.1037/0278-6133.24.5.443]

13. Antoniou AC, Hardy R, Walker L, et al. Predicting the likelihood of carrying a BRCA1 or BRCA2 mutation Validation of BOADICEA, BRCAPRO, IBIS, Myriad and the Manchester scoring system using data from UK genetics clinics. J Med Genet 2008;45(7):425-431. [http://dx.doi.org/10.1136/jmg.2007.056556]

4. Ardern-Jones A, Kenen R, Eeles R. Too much too soon? Patients' and health professionals' views concerning the impact of genetic testing at the time of breast cancer diagnosis in women under the age of 40. Eur J Cancer Care 2005;14(3):272-281. [http://dx.doi.org/10.1111/j.1365-2354.2005.00574.x]

15. Lobb EA, Barlow-Stewart K, Suthers G, et al. Treatment focused DNA testing for newly diagnosed breast cancer patients: Some implications for clinical practice. Clin Genet 2010;77(4):350-354. [http:// dx.doi.org/10.1111/j.1399-0004.2009.01307.x]

16. Zilliacu E, Meiser B, Gleeson M, et al. Are we being overly cautious? A qualitative inquiry into experiences and perceptions of treatment-focused germline BRCA genetic testing amongst women recently diagnosed with perceptions of treatment-focused germline BRCA genetic testing amongst women recently diagnosed with
breast cancer-Support Care Cancer 2012:20(11):2949-2958. [http///dx.doi.org/10.1007/s00520-012-1427-6]

17. Basro S, Apffelstaedt JP. Breast cancer in young women in a limited-resource environment. World J Sur Basro S, Apffelstaedt JP. Breast cancer in young women in a limited-res

2010;34(7):1427-1433. [http://dx.doi.org/10.1007/s00268-009-0299-5]
18. Fackenthal JD, Olopade, O.I. Breast cancer risk associated with BRCA1 and BRCA2 in diverse populations. Nat Rev Cancer 2007;7(12):937-949. [http://dx.doi.org/10.1038/nrc2054]

Accepted 19 June 2013. 